\title{
A Survey on Beam Steering Techniques in Printed Antennas
}

\author{
https://doi.org/10.3991/ijes.v9i2.21691 \\ Muhammad Irshad Khan ${ }^{(凶)}$, Muhammad Anab \\ University of Engineering \& Technology, Peshawar, Pakistan \\ m.i.khan@uetpeshawar.edu.pk \\ Muhammad Kabir Khan \\ Southwest Jiaotong University, Chengdu, China \\ Saeed-Ur-Rahman \\ Nanjing University of Aeronautics and Astronautics, Nanjing, China \\ Aamir Sultan \\ National University of Science \& Technology, Islamabad, Pakistan
}

\begin{abstract}
Beam steering antennas is essential part of various WCN (Wireless Communication Networks) such as radar and satellite communication. The increasing demand of high data rate, high SNR (Signal to Noise Ratio) and high gain, therefore pattern reconfigurable antennas are required to improve such properties. Beam steering is the technique of changing the main lobe direction of radiation. The constructive and distractive interference are used to steer the lobe in specific direction. Beam steering is necessary in various communications such as localization, satellite, tracking system and airborne application. Beam steering antenna decreases interference and power consumption and also increases directivity and gain. Beam steering antenna transmit and receive signals in specific direction. Beam steering antenna decreases interference and power consumption and also increases directivity and gain. The researchers are interested to find optimal beam steering solution for single and multi-point application. In past various techniques are used to achieve beam reconfigurable antennas. This paper presents, beam steering principle and their theory, beam steering techniques such as; Mechanical Steering, Beamforming, Switching Pin Diodes, Reflector and array antenna (Reflectarray), Parasitic Steering, Phase Shifters, Switched Beam Antennas, Metamaterial Antennas, Traveling Wave Antennas, Retro-directive Antennas, Integrated Lens Antennas (ILAS), merits and demerits and comparison among various techniques.
\end{abstract}

Keywords - Signal to Noise Ratio, Beam Steering Antennas, Directivity, Gain 


\section{Introduction}

Beam steering is the technique of changing the main lobe direction of radiation. The constructive and distractive interference are used to steer the lobe in specific direction. Beam steering is necessary in various communications such as localization, satellite, tracking system and airborne application [1-4]. Beam steering is used to differentiate the message signal from interference waves and steer the transmitted power in specific direction. Fig. 1 if the base station is omni-directional and needs only Device-2 signal, base station can receive all three signal simultaneously due its radiation pattern and cause interference problem. To avoid such problem reconfigurable pattern is used to focus the beam in the direction of Device-2 and avoid remain two signals [5]. Beam steering antenna decreases interference and power consumption and also increases directivity and gain. The researchers are interested to find optimal beam steering solution for single and multi-point application.

In this article, various techniques are discussed which is used for beam steering in past. In section 2 various techniques for beam steering are discussed which is already used in various applications, and in last section the past and present situation of improvement are concluded briefly. Beam steering is depicted in Fig. 1.

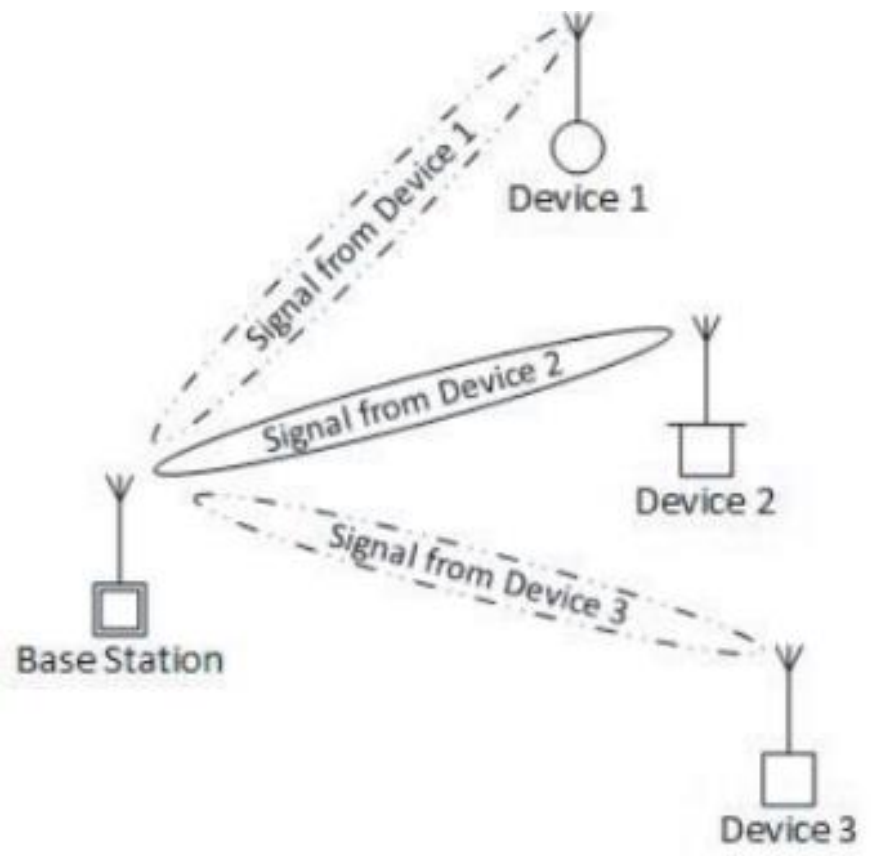

Fig. 1. Beam steering illustration 


\section{Beam Steering Techniques}

Various techniques are used for beam steering in last decade including:

a) Mechanical steering

b) Beamforming

c) SwitchingpPin diodes

d) Reflector and array antenna (Reflectarray)

e) Parasitic steering

f) Phase shifters

g) Switched beam antennas

h) Metamaterial antennas

i) Traveling wave antennas

j) Retro-directive antennas

k) Integrated Lens Antennas (ILAS)

\subsection{Mechanical steering}

Mechanical steering is manual method to change the direction of pattern. It is also difficult due to various factor such as size of antenna, weight and atmosphere change. In now-a-days mechanical steering uses MEMS (Micro-Electro-Mechanical System) devices, they reduced the losses and also improve the speed of scanning as compared to manual steering [6]. Mechanical steering is used to maintain the gain and show flexibility in range of steering [7]. However mechanical steering is implemented in very static environment, rotation phenomenon is main failure of such system [8]. To solve these problems various other electronics techniques are used.

\subsection{Beamforming}

Beam forming is the techniques to combine the wave of each element of array to form high directive beam of radiation. Beam forming is mainly used in alignment of signal phases come from various array elements and form high directive beam in desired direction. Beam forming is achieved to give time delay to every signal radiated from each element of array [9]. Special type of filters is used to form pencil beam to receive wave from specific area and avoid interference problem [10]. There are three types of beam forming such as Analogue, Digital and Hybrid beamforming.

In analogue beam forming the signal achieved from array is fed to low noise amplifier and then passed through phase shifter to give time delay to each signal. The time delay signal is passed through beam former to achieve resultant beam. This method is easy as compared to digital forming [11]. The architecture of analogue beam forming is depicted in Fig. 2. 


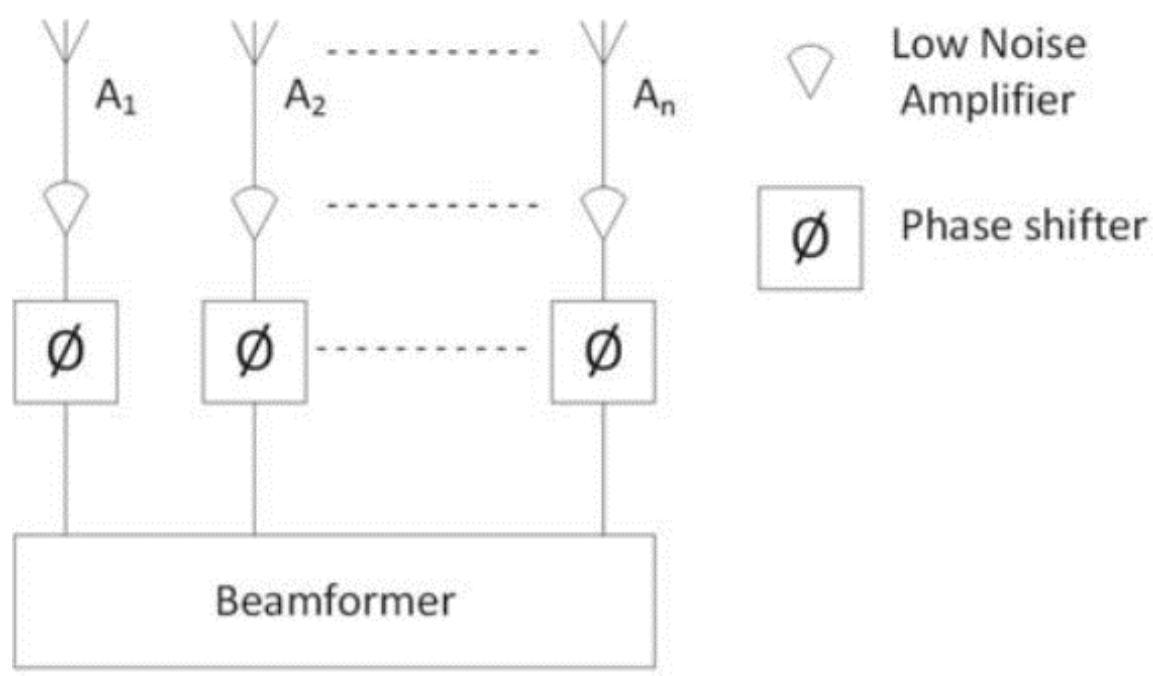

Fig. 2. Architecture of analogue beam forming

In digital beam forming the radiated signal from each element of array is passed through A/D (Analogue to Digital) converter to sample the signals. The digital signal is fed to down converter to convert it to lower frequency signals (baseband signal). The low frequency signals then pass through channeliser to divide the signal in channels. The resultant signal is passed through beam former to achieve steer beam [9]. The architecture is given in Fig. 3.

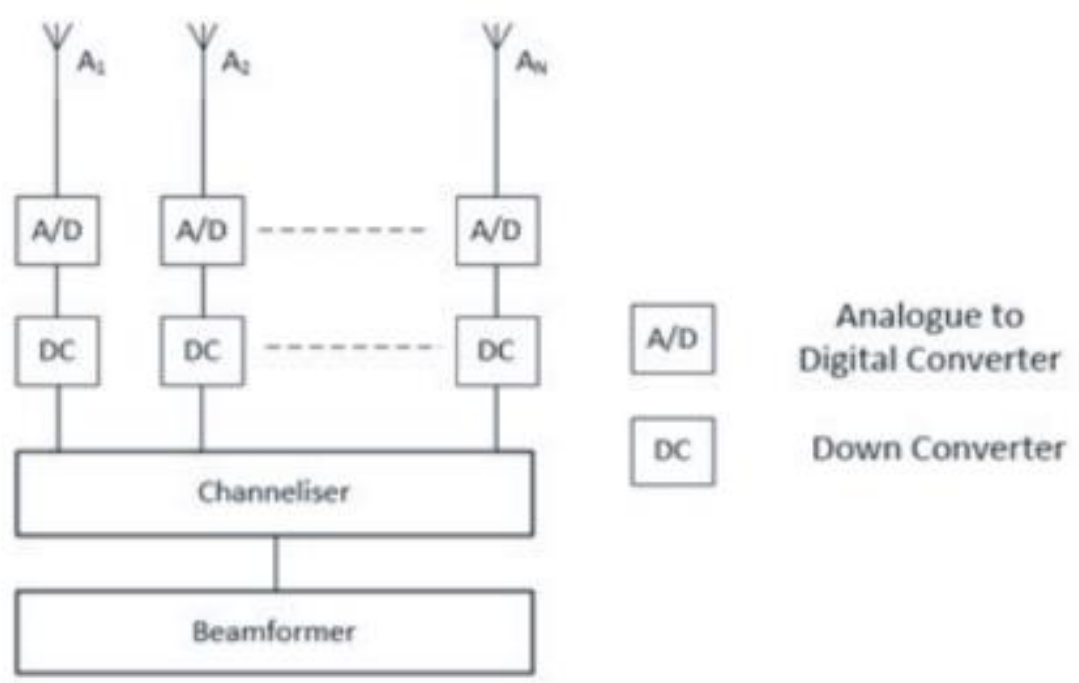

Fig. 3. Arcitecture of digital beam forming 
Hybrid beam forming is simply used to minimize the complexity and increase the performance of $\mathrm{D} / \mathrm{A}$ beamforming. In hybrid beamforming the analogue and digital beamforming is connected in series. The analogue beam forming is used for signal phases and digital beamforming is used for baseband signal processing [12]. The architecture of hybrid beamforming is illustrated in Fig. 4.

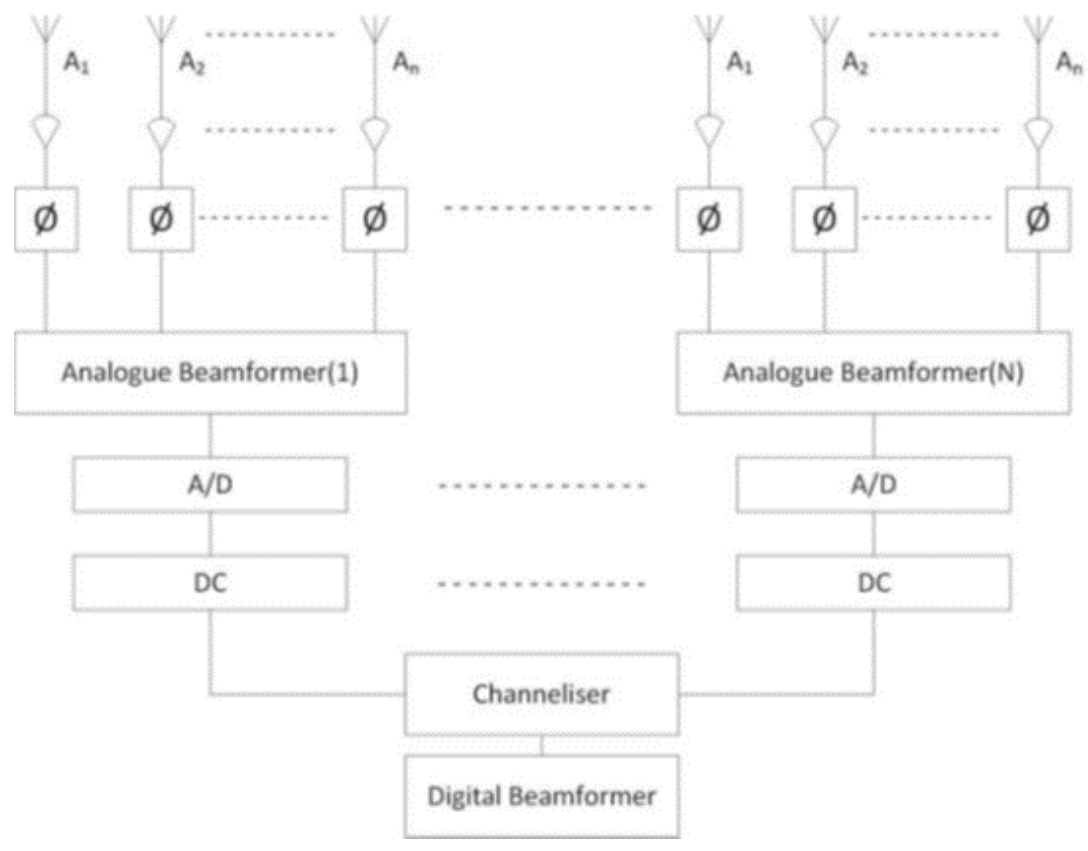

Fig. 4. Architecture of hybrid beam forming

\subsection{Switching pin diodes}

The pin diodes are also used to steer the beam of antenna. The pin diode works just like switch, these diodes are used between feed line and radiating patch or also used between ground and stub to control the pattern of antenna. The antenna is proposed for WiMAX (Worldwide Interoperability for Microwave Access) and WLAN (Wireless Local Area Network) application, the circular disc and four pin diodes are used to steer the pattern, the status of circular disc stub depend on the condition of pin diodes, it behave like ground or open ended to provide beam steering mechanism [13].

\subsection{Reflector and array antenna (Reflectarray)}

Reflector and antenna array are combined to achieve reconfigurable beam. In this technique the reflector is used to redirect the field to point of interest and antenna array is used to direct the incident signals, in this technique the phases are predefined to each element of array [14]. The phases are given to all elements through phase shifter or 
arrange the size and shape of each element of array [15]. The reflectarray mechanism is shown in Fig. 5.

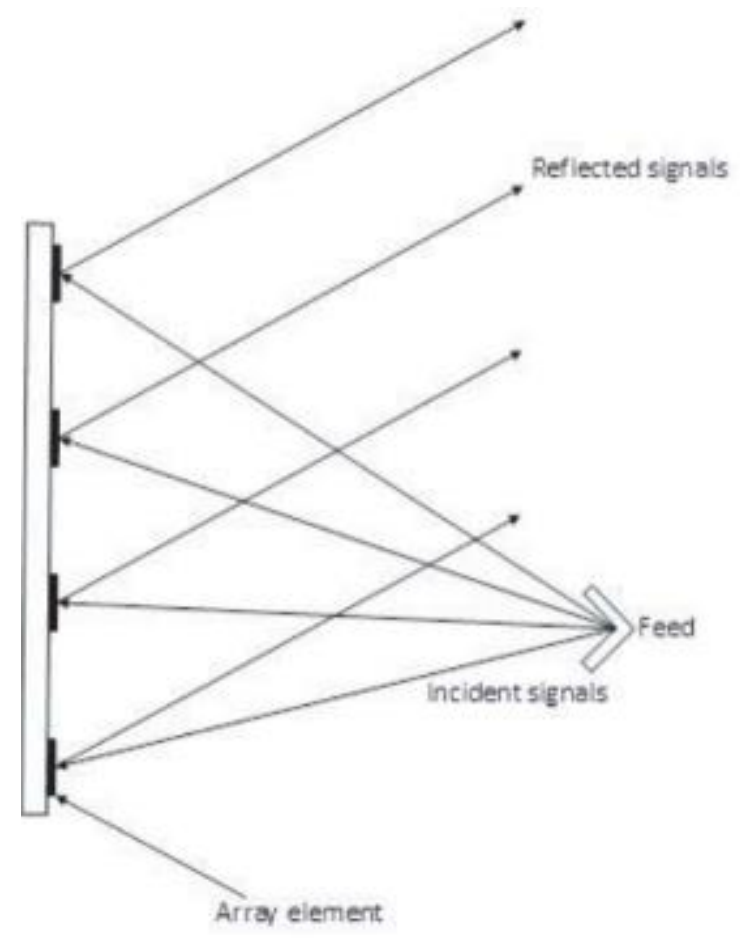

Fig. 5. Reflect-array mechanism

\subsection{Parasitic steering}

The parasitic element is a metallic strip which is not direct connected to electromagnetic power but exciting through coupling. Parasitic strip is passive element. Yagi antenna is suitable example of parasitic elements, depicted in Fig. 6. There are two types of parasitic elements such as directors and reflectors. Director is placed in front of driven element while reflector is placed behind driven elements. The directors increased the directivity of antenna, but if the directors are increased up to some limits, the electromagnetic coupling is decrease and there will be no effect on directivity. Various techniques are used for parasitic steering such as ESPAR (Electrically Steerable Passive Array Radiators), disk-loaded monopole array antennas and CSPA (Circular Switched Parasitic Array) [16-18]. The ground and parasitic element is connected through switch, when parasitic is connected to ground it behaves like reflector and behave like director in case of open circuit [16]. 


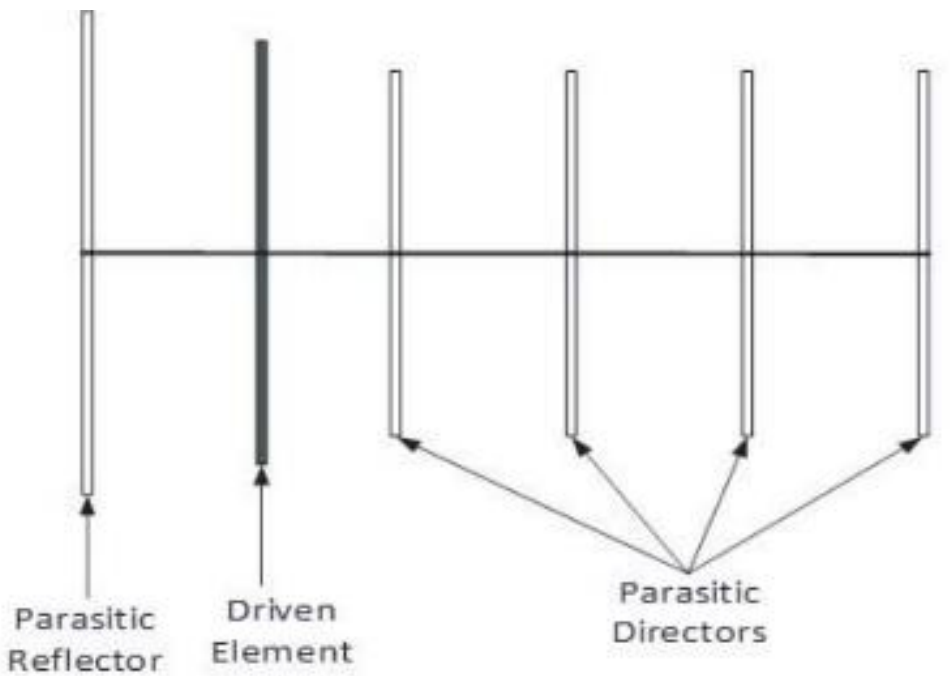

Fig. 6. Illustration of Yagi antenna

\subsection{Phase shifters}

Phase shifters are implemented to give time delay to each element signal of array. Phase shifter is used in analogue and hybrid beamforming. In analogue beamforming the signal achieved from array is fed to low noise amplifier and then passed through phase shifter to give time delay to each signal. The time delay signal is passed through beam former to achieve resultant beam. Special type of filters is used to form pencil beam to receive wave from specific area and avoid interference problem [10].

\subsection{Switched beam antennas}

In switched beam antennas technique, the antennas are arranged in circular pattern to cover desired angle [7]. If the single antenna element did not cover the desired range then the same antenna elements are arranged in circle to cover the range of interest, depicted in Fig. 7. Each antenna element covered very small section which is contributed to overall angle range. Switched beam antennas are nearly similar technique to ILAs (Integrated Lens Antennas), but in ILAs the antenna elements are arrange in straight line.

In Fig. 7, the eight elements are placed to cover the range which is very costly and insufficient solution. There is various problem in such system but the main issues are mutual interference, mutual coupling and also required the various feeding networks. 


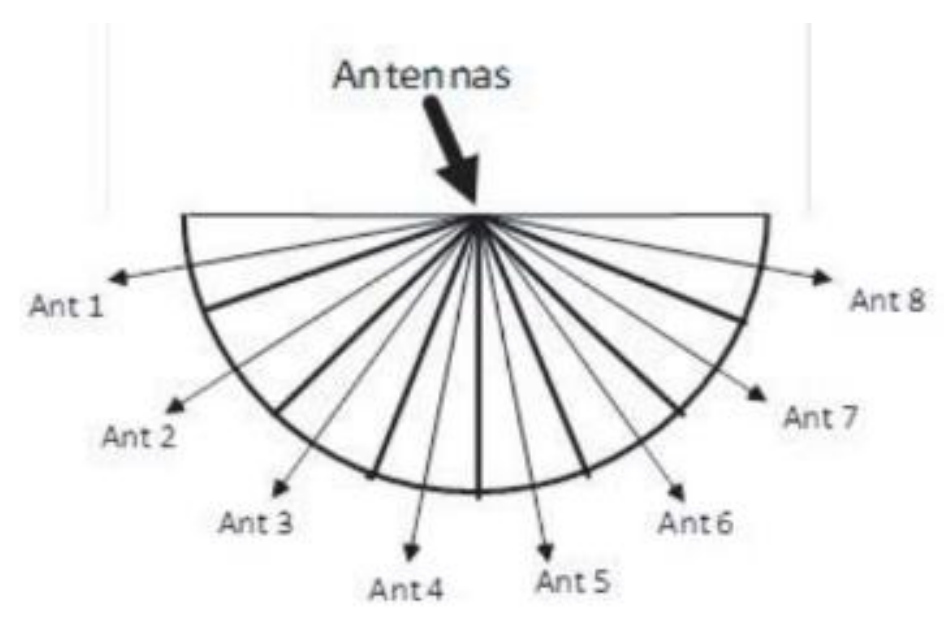

Fig. 7. Switched beam antennas schematics

\subsection{Metamaterial antennas}

Veselago gave the metamaterial concept for the first time in 1968 [19]. Metamaterial have various electromagnetic properties that cannot occurred in natural material structures [20]. The researchers take a lot of interest in last decade due to its permeability and permittivity, the permeability and permittivity of metamaterial exhibit both negative and positive value which is change according to their specification [21]. The metamaterial used widely in beam steering antenna because its radiation pattern are reconfigure by use of active element like transistors and diodes. The metamaterial saves the system from complex network like phase shifter (discussed in beamforming) and feeding networks (discussed in switched beam antennas).

In metamaterial the beam steering occurred due to change in refractive index and refractive index change with change in permeability and permittivity of material. The permeability and permittivity are changed due change in capacitance of each cell. The metamaterial is used as FSS (Frequency Selective Surfaces) when place above the radiating patch and each cell is loaded through varactors. Leaky Wave Antenna is designed by using metamaterial to achieve 40-degree steering [20]. The author proposed the metamaterial with $30^{\circ}$ steering and $12.5^{\circ}$ beam width, which is very limited steering [22]. The author proposed a wide steering about $\pm 56^{\circ}$ by using of multiple antennas with various positions, each element have their own metamaterial surface [23].

The metamaterial is also behaving like a lens to implement beam steering. The phenomenon is nearly related to ILAs, the metamaterial is placed on radiating patch as lens. The refractive index is changed with the help of active elements such as transistor and diodes in metamaterial, this phenomenon used in [24]. The authors achieve $\pm 30^{\circ}$ of beam steering. The author presented theoretical background about beam steering ranges, when metamaterial is used as lens [25]. There is no beam steering ranges achieved in measurements and simulation. 


\subsection{Traveling wave antennas}

There are two types of antenna in term of waves, standing wave and traveling wave antenna. Standing wave antenna is also known as resonant antenna. In standing wave antenna the pattern is created due to reflected wave from the open end of conductor and zero current is passed through conductor this phenomenon occur due to mismatching [26]. In TWA (Traveling Wave Antennas) the radiating element is fully matched at open end, so current is moving and radiation pattern is form in form of current and voltage. There are two main types of TWA [26].

Surface wave antenna: Surface wave antenna is also called slow wave antenna because in free space the speed of light is greater than the phase velocity of guide wave (Slow phase velocity than light speed). This type of antenna is also resonate at discontinuities due to its slow speed. Discontinuities are feeding port and end of radiating elements. Its radiation pattern is not highly directive due its side lobes levels. The antenna size determined the location of main beam [27]. Helixes and corrugated conductors are two main example of surface wave antenna.

Leaky wave antenna: Leaky wave antenna is also called fast wave antenna because in free space the speed of light is less than the phase velocity of guide wave (Fast phase velocity than light speed). LWA (Leaky Wave Antenna) is radiating continuously due to its high speed. Two variables is used to control the angle and beam width of beam. The phase constant control the angle of beam and attenuation constant control the beam width of the beam. The frequency changed the phase constant and phase constant change the angle of beam [27].

TWA is used mostly for wideband application because it radiates on all frequencies in specific bandwidth [28]. Beam steering is also implemented for fixed frequencies; there are various methods such as dual feeding points, parasitic effect or Slot loading and using materials with adjustable properties. In dual feeding techniques the antenna is fed at two points due to which the beam is mirrored at vertical plane [29-30].

In parasitic effect or Slot loading, the array patch is used around LWA which is away from antenna with small distance, switches is used to short ground to patch. The frequency is steer accordingly with the change in states of switches [31]. The author achieves $37^{\circ}$ of beam steering, when all patch elements are OFF or ON [32].

In using materials with adjustable properties, metamaterial and graphene are combined to steer the beam of LWA at predefined frequency. The conductivity of graphene is tuned which is used as a ground plane to steer the beam of LWA [33]. The beam is also steer due to change in phase gradient which is implemented in [34].

\subsection{Ret-directive antennas}

Retro-directive is the phenomenon, to redirect anything to specific position. In antenna terminology, any antenna that poses the ability to redirect the electromagnetic wave from backward to specific position (origin) is known as retro-directive antenna. In this type of antenna used reflectors, in radar the light wave is directed towards origin by mean of reflectors [35]. The researchers are interested in retro-directive antenna for beam forming because it is easy to design as compared to beamforming. There are two 
ways to implement retro-directive array antenna such as phase conjugating mixers architecture and Van Atta array [36].

In phase conjugating mixers the phases are reversed through mixers and local oscillators instead of pairs antennas [35]. In this technique the mixers are pumped through oscillators to double the frequency, this design is simple in implementation. Phases are also reversed through digital and phase detection methods, these methods reduced complexity and costs [37]. The structure of phase conjugate mixer is depicted in Fig. 8(a).

In Van Atta array, the pairs of antennas are used which is place with equal distances from origin of array. In these techniques one antenna receives signal remain antenna resonate the signal to source. The transmission line principle is used to reverse phases which need specific length between the pairs [36]. The structure of van Atta array is depicted in Fig. 8(b).

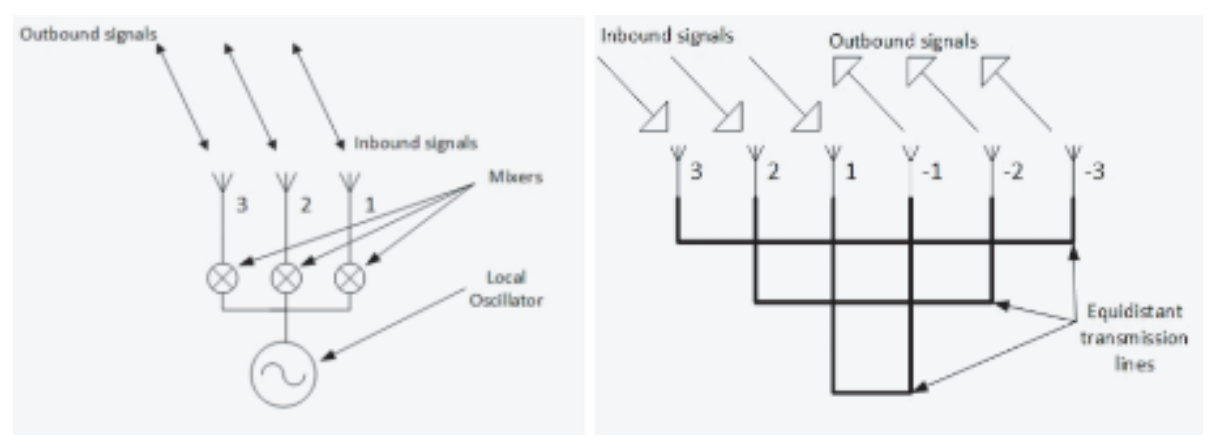

a) Phase conjugating mixer

b) Van Atta array

Fig. 8. The architecture of Retro-directive array

\subsection{Integrated lens antennas}

Rutledge proposed concept for the first time to integrate the antenna with lens [38]. The architecture is depicted in Fig. 9. From Fig. 9 the radiating elements (antennas) are placed with same distance denoted by " $d$ " and elliptical lens is used to manage the radiating wave towards focal point [39-43]. The special techniques are used to set the lens the radiating signal of each element is passed through focal point, the radiating element is radiates one by one to steer the beam for various fixed frequency, the radiating elements are controlled through switches [39,42]. The ILAs achieved high directional beam as compared to phase array antennas, the ILAs used switch which is very simple and low profile as compared to phase shifter [40].

The author proposed the antenna for 52 and $68 \mathrm{GHz}$ with the beam steering angle of \pm 35 and $\pm 22^{\circ}$, the radius of the lens is $7.5 \mathrm{~mm}$ and the gain is $18.4 \mathrm{dBi}$ [43]. To achieved large steering angle the offset among radiating elements increased, by increasing offset gain scan loss also increased. Gain scan loss is the difference between gain of deviated beam direction and reconfigurable beam direction. The gain scan loss depends on ratio 
of offsets between antenna and radius of lens [44]. In fabrication of lenses various losses occurred, these types of losses depend on material used in fabrication [45]. In last decade, the researchers try to use metamaterial in ILAs for improvements.

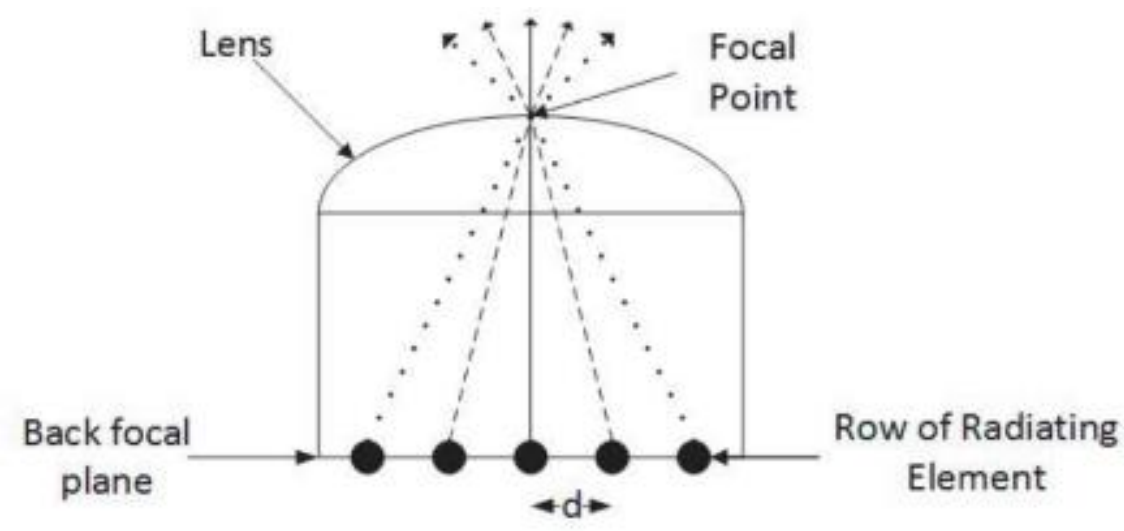

Fig. 9. Illustration of Integrated Lens Antennas

\section{Conclusion}

In this article, the techniques used for beam steering are discussed in detail, the limitation are also discussed. Beam steering antennas is essential part of various wireless communication networks such as radar and satellite communication. The increasing demand of high data rate, high signal to noise ratio and high gain, therefore pattern reconfigurable antennas are required to improve such properties. Beam steering antenna decreases interference and power consumption and also increases directivity and gain. Beam steering antenna transmits and receives signals in specific direction. Beam steering is the technique of changing the main lobe direction of radiation. The constructive and distractive interference are used to steer the lobe in specific direction. Beam steering is necessary in various communications such as localization, satellite, tracking system and airborne application. Beam steering is used to differentiate the message signal from interference waves and steer the transmitted power in specific direction.

Mechanical steering is manual method to change the direction of pattern. It is also difficult due to various factors such as size of antenna, weight and atmosphere change. In now-a-days mechanical steering uses MEMS devices, they reduced the losses and also improve the speed of scanning as compared to manual steering. Beam forming is the techniques to combine the wave of each element of array to form high directive beam of radiation. Beam forming is mainly used in alignment of signal phases come from various array elements and form high directive beam in desired direction. The pin diodes are also used to steer the beam of antenna. The pin diode works just like switch, these diodes are used between feed line and radiating patch or also used between ground and stub to control the pattern of antenna. Reflector and antenna array are combined to achieve reconfigurable beam. In this technique the reflector is used to redirect the field 
to point of interest and antenna array is used to direct the incident signals. The parasitic element is a metallic strip which is not direct connected to electromagnetic power but exciting through coupling. Parasitic strip is passive element. Yagi antenna is suitable example of parasitic elements. Phase shifters are implemented to give time delay to each element signal of array. Phase shifter are used in analogue and hybrid beamforming.

In switched beam antennas technique, the antennas are arranged in circular pattern to cover desired angle. If the single antenna element did not cover the desired range, then the same antenna elements are arranged in circle to cover the range of interest. Each antenna element covered very small section which is contributed to overall angle range. Switched beam antennas is nearly similar technique to ILAs, but in ILAs the antenna elements are arrange in straight line. Veselago gave the metamaterial concept for the first time in 1968. Metamaterial have various electromagnetic properties that cannot occurred in natural material structures. The researchers take a lot of interest in last decade due to its permeability and permittivity, the permeability and permittivity of metamaterial exhibit both negative and positive value which is change according to their specification. The metamaterial used widely in beam steering antenna because its radiation pattern is reconfigured by use of active element like transistors and diodes. In TWA the radiating element is fully matched at open end, so current is moving and radiation pattern is form in form of current and voltage. There are two main types of TWA such as surface wave antenna and leaky wave antenna. Retro-directive is the phenomenon, to redirect anything to specific position. In antenna terminology, any antenna that poses the ability to redirect the electromagnetic wave from backward to specific position (origin) is known as retro-directive antenna. In this type of antenna used reflectors, in radar the light wave is directed towards origin by mean of reflectors. In ILAs the radiating elements (antennas) are placed with same distance denoted by " $d$ " and elliptical lens is used to manage the radiating wave towards focal point. The special techniques are used to set the lens the radiating signal of each element is passed through focal point, the radiating element is radiating one by one to steer the beam for various fixed frequency, the radiating elements are controlled through switches.

\section{Conflicts of interest}

The authors declare that there are no conflicts of interest regarding the publication of this paper.

\section{$5 \quad$ References}

[1] T.D. Bui, B.H. Nguyen, Q.C. Nguyen, and M.T. Le, "Design of Beam Steering Antenna for Localization Applications", IEEE International Symposium on Antennas and Propagation, pp. 956-957, October, 2016.

[2] I. Kaplan, I. Marinov, A. Gal, V. Peshlov, M. Gachev, V. Boyanov, and B. Marinov, "Electronically Beam Steerable Antennas for Broadband Satellite Communications", IEEE 8th European Conference on Antennas and Propagation, pp. 2450-2454, April, 2014. https://doi.org/10.1109/eucap.2014.6902313 
[3] H. Aliakbarian, E. Van der Westhuizen, R. Wiid, V. Volski, R. Wolhuter, G.A. Vandenbosch, and P. Coppin, "A Digitally Beam-Steerable Antenna Array System for PositioningBased Tracking Applications", IEEE Antennas and Propagation Magazine, Volume 55, No. 6, pp. 35-49, 2013. https://doi.org/10.1109/map.2013.6781704

[4] H. Aliakbarian, E. Van der Westhuizen, R. Wiid, V. Volskiy, R. Wolhuter, and G.A. Vandenbosch, "An Efficient Beam Steerable Antenna Array Concept for Airborne Applications", Radio Engineering, Volume 23, No. 1, April, 2014.

[5] I. Uchendu, and J.R. Kelly, "Survey of Beam Steering Techniques Available for Millimeter Wave Applications", Electromagnetics Research, Volume 68, pp. 35-54, 2016. https://doi.org/10.2528/pierb16030703

[6] J. Chiao, Y. Fu, I. M. Chio, M. DeLisio, and L.Y. Lin, "MEMS Reconfigurable VEE Antenna", IEEE MTT-S International Microwave Symposium, Volume 4, pp. 1515-1518, 1999. https://doi.org/10.1109/mwsym.1999.780242

[7] C.W. Baek, S. Song, C. Cheon, Y. Kim, and Y. Kwon, "2D Mechanical Beam Steering Antenna Fabricated Using MEMS Technology", IEEE MTT-S International Microwave Symposium, Volume 1, 2001. https://doi.org/10.1109/mwsym.2001.966873

[8] D. Rodrigo, L. Jofre, and B.A. Cetiner, "Circular Beam-Steering Reconfigurable Antenna with Liquid Metal Parasitics", IEEE Transactions on Antennas Propagation., Volume 60, No. 4, pp. 1796-1802, 2012. https://doi.org/10.1109/tap.2012.2186235

[9] K. Zarb-Adami, A. Faulkner, J.G.B. De Vaate, G.W. Kant, and P. Picard, "Beamforming Techniques for Large-N Aperture Arrays", IEEE International Symposium on Phased Array System Technology, pp. 883-890, 2010. https://doi.org/10.1109/array.2010.5613258

[10] B.D. Van Veen, and K.M. Buckley, "Beamforming: A Versatile Approach to Spatial Filtering”, IEEE ASSP Magazine, Volume 5, pp. 4-24, 1988. https://doi.org/10.1109/53.665

[11] H. Steyskal, "Digital Beamforming", 18th European Microwave Conference, pp. 49-57, 1988. https://doi.org/10.1109/euma.1988.333796

[12] S. Han, I. Chih-Lin, Z. Xu, and S. Wang, "Reference Signals Design for Hybrid Analog and Digital Beamforming", IEEE Communication Letter, Volume 18, No. 7, pp. 1191-1193, 2014. https://doi.org/10.1109/lcomm.2014.2317747

[13] M.S. Alam, and A.M. Abbosh, "Beam-Steerable Planar Antenna Using Circular Disc and Four PIN-Controlled Tapered Stubs for WiMAX and WLAN Applications", IEEE Antennas and Wireless Propagation Letters, Volume 15, pp. 980-983, 2016. https://doi.org/10.1109/lawp.2015.2489684

[14] A.V. Raisanen, J. Ala-Laurinaho, D. Chicherin, Z. Du, A. Generalov, A. Karttunen, D. Lioubtchenko, J. Mallat, A. Tamminen, and T. Zvolensky, "Beam-Steering Antennas at Millimeter Wavelengths", Proceedings of 5th Global Symposium on Millimeter-Waves, pp. 170-173, May, 2012. https://doi.org/10.1109/gsmm.2012.6314028

[15] D. Antar, and Y. Guha, "Microstrip and Printed Antennas: New Trends, Techniques and Applications", John Wiley \& Sons, Hoboken, NJ, USA, 2010. https://doi.org/10.1002/ 9780470973370

[16] H. Kawakami, and T. Ohira, "Electrically Steerable Passive Array Radiator (ESPAR) Antennas", IEEE Antennas Propagation Magazine, Volume 47, No. 2, pp. 43-50, April, 2005. https://doi.org/10.1109/map.2005.1487777

[17] M.R. Kamarudin, P.S. Hall, F. Colombel, and M. Himdi, "Electronically Switched Beam Disk Loaded Monopole Array Antenna", Progress in Electromagnetics Research, Volume 101, pp. 339-347, 2010. https://doi.org/10.2528/pier10010808

[18] R. Vilar, R. Czarny, M.L. Lee, B. Loiseaux, M. Sypek, M. Makowski, C. Martel, T. Crepin, F. Boust, R. Joseph, K. Herbertz, T. Bertuch, and J. Marti, "Q-Band Millimeter-Wave Antennas: An Enabling Technology for Multigigabit Wireless Backhaul”, IEEE Microwave Magazine, Volume 15, No. 4, pp. 121-130, June, 2014. https://doi.org/10.1109 $\underline{\mathrm{mmm} .2014 .2308769}$ 
[19] V.G. Veselago, "The Electrodynamics of Substances with Simultaneously Negative Values of $\varepsilon$ and $\mu$ ", Soviet Physics Uspekhi, Volume 10, No. 4, pp. 509-514, January-February, 1968. https://doi.org/10.1070/pu1968v010n04abeh003699

[20] A. Symeonidou, and K. Siakavara, "A Novel Microstrip Antenna Array with MetamaterialBased Electronic Beam Steering at $2.4 \mathrm{GHz}$ ", Progress in Electromagnetics Research-C, Volume 38, pp. 27-42, 2013. https://doi.org/10.2528/pierc13020405

[21] T. Jiang, Z. Wang, D. Li, J. Pan, B. Zhang, J. Huangfu, Y. Salamin, C. Li, and L. Ran, "LowDC Voltage-Controlled Steering-Antenna Random Utilizing Tunable Active Metamaterial”, IEEE Transactions on Microwave Theory Technology, Volume 60, No. 1, pp. 170-178, January, 2012. https://doi.org/10.1109/tmtt.2011.2171981

[22] H. Li, D. Ye, F. Shen, B. Zhang, Y. Sun, W. Zhu, C. Li, and L. Ran, "Reconfigurable Diffractive Antenna Based on Switchable Electrically Induced Transparency", IEEE Transactions on Microwave Theory Technology, Volume 63, No. 3, pp. 925-936, March, 2015. https://doi.org/10.1109/tmtt.2015.2393862

[23] A. Dadgarpour, B. Zarghooni, B.S. Virdee, and T.A. Denidni, "Beam-Deflection Using Gradient Refractive-Index Media for 60-GHz NND-Fire Antenna", IEEE Transactions on Antennas Propagation, Volume 63, No. 8, pp. 3768-3774, August, 2015. https://doi.org/ 10.1109/tap.2015.2438396

[24] F. Sun, Y. Ma, and S. He, "Two Beam Steering Lenses Enabled by Metamaterials", IEEE MTT-S International Microwave Workshop Series on Advanced Materials and Processes for RF and THz Applications, Suzhou, 2015. https://doi.org/10.1109/imws-amp.2015. 7325049

[25] Z.N. Wu, W.X. Tang, and T.J. Cui, “A Beam-Steerable Metamaterial Lens Using Varactor Diodes", IEEE MTT-S International Microwave Workshop Series on Advanced Materials and Processes for RF and THz Applications, Suzhou, 2015. https://doi.org/10.1109/imwsamp.2015.7324902

[26] C.A. Balanis, “Antenna Theory: Analysis and Design”, $3^{\text {rd }}$ Edition, John Wiley \&Sons Inc., Canada, 2005.

[27] F. Frezza, "Introduction to Traveling-Wave Antennas", European School of Antennas, pp. $1-10,2006$.

[28] D.K. Karmokar, and K.P. Esselle, "Periodic U-Slot-Loaded Dual-Band Half-Width Microstrip Leaky Wave Antennas for Forward and Backward Beam Scanning”, IEEE Transactions on Antennas Propagation, Volume 63, No. 12, pp. 5372-5381, December, 2015. https://doi.org/10.1109/tap.2015.2490252

[29] E. Topak, J. Hasch, C. Wagner, and T. Zwick, "A Novel Millimeter-Wave Dual-Fed Phased Array for Beam Steering", IEEE Transactions on Microwave Theory Technology, Volume 61, No. 8, pp. 3140-3147, August, 2013. https://doi.org/10.1109/tmtt.2013.22 $\underline{67935}$

[30] M. Khalil, M. Kamarei, J. Jomaah, and H. Ayad, "Compact SIW Leaky Wave Antenna”, $3^{\text {rd }}$ International Conference on Technological Advances in Electrical, Electronics and Computer Engineering, pp. 124-129, April, 2015. https://doi.org/10.1109/taeece. 2015.7113612

[31] D.K. Karmokar, K.P. Esselle, and S.G. Hay, "A Microstrip Leaky-Wave Antenna with Two Symmetrical Beams Towards Sides for Fixed-Frequency Dual-Beam Scanning", $16^{\text {th }}$ International Symposium on Antenna Technology and Applied Electromagnetics, July 13-16, 2014. https://doi.org/10.1109/antem.2014.6887724

[32] D.K. Karmokar, K.P. Esselle, and S.G. Hay, "Shifting the Fixed-Frequency Beam Scanning Range of a Leaky-Wave Antenna by Slot Loading", IEEE-APS Topical Conference on Antennas and Propagation in Wireless Communications, pp. 640-643, August 3-9, 2014. https://doi.org/10.1109/apwc.2014.6905576 
[33] X. Wang, W. Zhao, J. Hu, and W. Yin, "Reconfigurable Terahertz Leaky-Wave Antenna Using Graphene-Based High-Impedance Surface", IEEE Transactions on Nanotechnology, Volume 14, No. 1, pp. 62-69, January, 2015. https://doi.org/10.1109/tnano.2014.2365205

[34] A. Ghasemi, S.N. Burokur, A. Dhouibi, and A. de Lustrac, "Phase-Gradient Metasurfaces for Beam Steerable Antennas", International Workshop on Antenna Technology: Small Antennas, Novel EM Structures and Materials, and Applications, pp. 191-194, March 4-6, 2014. https://doi.org/10.1109/iwat.2014.6958635

[35] y.-C. Guo, X.-W. Shi, and L. Chen, "Retrodirective Array Technology", Progress in Electromagnetics Research-B, Volume 5, pp. 153-167, 2008. https://doi.org/10.2528/pierb 08021704

[36] R.Y. Miyamoto, and T. Itoh, "Retrodirective Arrays for Wireless Communications", IEEE Microwave Magazine, Volume 3, No. 1, pp. 71-79, March, 2002. https://doi.org/10.1109/ 6668.990692

[37] L. Chen, Y.C. Guo, X.W. Shi, and T.L. Zhang, "Overview on the Phase Conjugation Techniques of the Retrodirective Array", International Journal of Antennas Propagation, 2010.

[38] T.H. Buttgenbach, "Improved Solution for Integrated Array Optics in Quasi-Optical mm and Sub-mm Receivers: The hybrid Antenna", IEEE Transactions on Microwave Theory Technology, Volume 41, No. 10, pp. 1750-1761, October, 1993. https://doi.org/10.1109 $\underline{122.247920}$

[39] A.V. Raisanen, J. Ala-Laurinaho, K. Haneda, J. Jarvelainen, A. Karttunen, M. Kyro, V. Semkin, A. Lamminen, and J. Saily, "Studies on E-Band Antennas and Propagation", Loughborough Antennas \&Propagation Conference, pp. 176-180, November, 2013. https://doi.org/10.1109/lapc.2013.6711876

[40] A. Artemenko, A. Maltsev, R. Maslennikov, A. Sevastyanov, and V. Ssorin, "Beam Steerable Quartz Integrated Lens Antenna for 60GHz Frequency Band", Proceedings of 5th European Conference Antennas Propagation, pp. 758 - 762, April, 2011. https://doi.org/10.1109/eucap.2016.7481774

[41] J. Ala-Laurinaho, A. Karttunen, J. Saily, A. Lamminen, R. Sauleau, and A.V. Raisanen, "MMwave Lens Antenna with an Integrated LTCC Feed Array for Beam Steering" Proceedings of $4^{\text {th }}$ European Conference Antennas Propagation, 2010. https://doi.org/10.1109 leucap.2012.6205892

[42] A. Artemenko, A. Mozharovskiy, A. Maltsev, R. Maslennikov, A. Sevastyanov, and V. Ssorin, "2D Electronically Beam Steerable Integrated Lens Antennas for mm-Wave Applications", 42 ${ }^{\text {nd }}$ European Microwave Conference, pp. 213-216, November, 2012. https:// doi.org/10.23919/eumc.2012.6459155

[43] A. Artemenko, A. Maltsev, A. Mozharovskiy, A. Sevastyanov, V. Ssorin, and R. Maslennikov, "Millimeter-Wave Electronically Steerable Integrated Lens Antennas for WLAN/WPAN Applications", IEEE Transactions on Antennas Propagation, Volume 61, No. 4, pp. 1665-1671, April, 2013. https://doi.org/10.1109/tap.2012.2232266

[44] D.F. Filipovic, G.P. Gauthier, S. Raman, and G.M. Rebeiz, "Off-Axis Properties of Silicon and Quartz Dielectric Lens Antennas", IEEE Transactions on Antennas Propagation, Volume 45, No. 5, pp. 760-766, May, 1997. https://doi.org/10.1109/8.575618

[45] T.A. Lam, D.C. Vier, J.A. Nielsen, C.G. Parazzoli, and M.H. Tanielian, "Steering Phased Array Antenna Beams to the Horizon Using a Buckyball NIM Lens", Proceedings of IEEE, Volume 99, No. 10, pp. 1755-1767, October, 2011. https://doi.org/10.1109 liproc.2011.2128290

\section{Authors}

Muhammad Irshad Khan received the B.Sc. (Hons.) degree in Electrical Electronic engineering from the Comsats Institute of Information Technology Abbottabad, 
Pakistan and the M.Sc. degree in Electrical engineering from the Capital University of Science and Technology, Islamabad, Pakistan in 2016. He is currently pursuing the $\mathrm{Ph} . \mathrm{D}$. degree in electrical engineering from the University of Engineering and Technology, Peshawar, Kohat Campus, Pakistan. His research interests include microwave and RF circuit design. UWB antenna design and analysis, MIMO antennas, Beam steering antennas and reconfigurable antenna design.

Muhammad Anab is working as Lab. Engineer in the Department of Electrical Engineering in University of Engineering and Technology Peshawar. He received his B.Sc. (Hons.) degree in Electrical Engineering from University of Engineering and Technology Peshawar in 2016 and the M.Sc. degree in Electrical engineering from the same University in 2019. He is also a member of iMicrowave and Antenna Research Groupî supervised by Dr. Muhammad Irfan Khattak and his research interests include DRA antenna design and analysis for 5G Applications, 5G Microstrip patch Antenna design and Graphene Based Terahertz antennas. m.anab@uetpeshawar.edu.pk

Muhammad Kabir Khan received the B.Sc. (Hons.) and MS degree in Computer engineering from the Comsats Institute of Information Technology Abbottabad, Pakistan. He is currently pursuing the Ph.D. degree in electrical engineering from School of Information Science \& Technology, Southwest Jiaotong University, Chengdu 611756, China. His research interests include UWB antenna design and analysis, MIMO antennas, Beam steering antennas and reconfigurable antenna design. kabirnawab@gmail.com

Saeed-Ur-Rahman received BS in Electronics Engineering from COMSAT University, Pakistan in 2013 and MS in Electronics Engineering from Capital University of Science and Technology (CUST), Pakistan in 2016. Recently, he is pursuing his PhD from College of Electronic \& Information Engineering, Nanjing University of Aeronautics and Astronautics (NUAA), Nanjing210016, China, under the supervision of Prof. Qunsheng Cao. His research interests include electromagnetics and antennas especially designing, and optimization of antenna array, micro-strip patch antennas, ultrawideband antennas, and meta-materials. saeed@nuaa.edu.cn

Aamir Sultan completed M.S degree in Electrical engineering from College of Electrical \& Mechanical Engineering, National University of Science \& Technology, Islamabad. His research interests include electromagnetics and antennas especially designing, micro-strip patch, DRA antenna design and analysis for 5G Applications. asultan17@ee.ceme.edu.pk

Article submitted 2021-02-02. Resubmitted 2021-03-13. Final acceptance 2021-03-16. Final version published as submitted by the authors. 